\title{
Nonfinite Axiomatizability of Shuffle Inequalities
}

\author{
Stephen L. Bloomªnd Zoltán Ésik ${ }^{\star \star}$ \\ Stevens Institute of Technology \\ Department of Computer Science \\ Hoboken, NJ 07030 \\ bloomegauss.stevens-tech.edu
}

\begin{abstract}
There is some set of inequations $t \leq t^{\prime}$ whose models are the algebras in the variety of ordered algebras generated by the algebras $\mathcal{L}_{\Sigma}=\left(P_{\Sigma}, \cdot, \otimes, 1\right)$, where $P_{\Sigma}$ consists of all subsets of the free monoid $\Sigma^{*}, B \cdot C=\{u v: u \in B, v \in C\}$, and $B \otimes C$ is the shuffle product of the two languages. We show that there is no finite set of such inequations.
\end{abstract}

\section{Introduction}

In a previous paper [4], the authors found concrete descriptions of the free algebras in varieties generated by languages equipped with various sets of operations all involving the shuffle operation. For example, we considered the variety generated by the structures $\mathcal{L}_{\Sigma}=\left(P_{\Sigma}, \cdot, \otimes, 1\right)$, where $P_{\Sigma}$ consists of all subsets of the free monoid $\Sigma^{*}, B \cdot C=\{u v: u \in B, v \in C\}$, and $B \otimes C$ is the shuffle product of the two languages. The free algebras in this variety are labeled series-parallel posets. The free algebras in the variety of ordered algebras generated by the structures $\left(\mathcal{L}_{\Sigma}, \subseteq\right)$ are again the labeled series-parallel posets equipped with an ordering we call the trace ordering. (In [7], labeled posets with the "subsumption order" were shown to be the free ordered algebras in a finitely axiomatizable variety.) "Closed subsets" of the labeled posets are the free algebras in the variety generated by the structures $\left(P_{\Sigma}, \cdot, \otimes,+,{ }^{*}, 0,1\right)$, where + is binary union and ${ }^{*}$ is the Kleene star operation. These results seem to show that two different models of parallelism are equivalent: the so-called language or interleaving model, and a pomset model.

What the result in [4] does not establish is precisely what the valid inequations or equations are in each of the varieties considered. One would like to have an

\footnotetext{
* Partially supported by the US-Hungarian Joint Fund under grant number 351.

** Partially supported by a grant of the National Foundation for Scientific Research of Hungary, the Alexander von Humboldt Foundation, and by the US-Hungarian Joint Fund under grant number 351 .
} 
axiomatic description of the set of valid equations and inequations, preferably via a simple finite set of axioms. In this paper, we consider the variety $\mathbf{L} \mathbf{g}_{\leq}$of ordered algebras generated by the language structures $\left(P_{\Sigma}, \cdot, \otimes, 1, \subseteq\right)$ and show that there is no finite set of inequations which axiomatize the inequations valid in $\mathbf{L} \mathbf{g} \leq$.

The axiomatization of the varieties generated by the language structures $\left(P_{\Sigma}, \cdot, \otimes\right.$, $+, 0,1)$ and $\left(P_{\Sigma}, \cdot, \otimes,+,{ }^{*}, 0,1\right)$ is considered in the paper [5].

\section{Review}

In this section, we review the necessary definitions and results from [4].

Definition 1. A bimonoid $\mathrm{M}=(M, \cdot, \otimes, 1)$ consists of a monoid $(M, \cdot, 1)$ and a commutative monoid $(M, \otimes, 1)$ with a common neutral element. A bimonoid morphism $\mathrm{M} \rightarrow \mathrm{M}^{\prime}$ is a function $M \rightarrow M^{\prime}$ which preserves the unit and the two binary operations.

Definition 2. An ordered bimonoid $(\mathrm{M}, \leq)$ is a bimonoid $(M, \cdot, \otimes, 1)$ whose underlying set $M$ is equipped with a partial ordering $\leq$ such that for all $x, y, a, b \in$ $M$,

$$
x \leq a, y \leq b \Rightarrow x \cdot y \leq a \cdot b \text { and } x \otimes y \leq a \otimes b .
$$

A morphism of ordered bimonoids is an order preserving bimonoid morphism.

Three motivating examples of ordered bimonoids are the language structures $\mathcal{L}_{\Sigma}:=\left(P_{\Sigma}, \cdot, \otimes, 1\right), \mathcal{R}_{\Sigma}:=\left(R_{\Sigma}, \cdot, \otimes, 1\right), \mathcal{F}_{\Sigma}:=\left(F_{\Sigma}, \cdot, \otimes, 1\right)$, where $P_{\Sigma}$ is the collection of all subsets, $R_{\Sigma}$ consists of the regular subsets, and $F_{\Sigma}$ consists of the finite subsets of the free monoid $\Sigma^{*}$. The ordering on each bimonoid is that of set inclusion. The set $B \cdot C$ is $\{u v: u \in B, v \in C\}$, and $B \otimes C$ is the shuffle product of languages:

$B \otimes C:=\left\{u_{1} v_{1} \cdots u_{n} v_{n}: u_{1} \cdots u_{n} \in B, v_{1} \cdots v_{n} \in C, u_{i}, v_{i} \in \Sigma^{*}, n \geq 0\right\}$

If $f: A \rightarrow B$ is a morphism of ordered bimonoids, we say $f$ is order-reflecting if $x f \leq y f \Leftrightarrow x \leq y$. Note that an order-reflecting morphism is necessarily injective. Say that the ordered bimonoid $A$ is an ordered subalgebra of the ordered bimonoid $B$ if there is an order-reflecting morphism $A \rightarrow B$. A variety $\mathcal{V}$ of ordered bimonoids is a collection of ordered bimonoids closed under products (ordered componentwise), ordered subalgebras and order preserving morphic images. Equivalently, (see [3]), a variety of ordered bimonoids is the collection of all ordered bimonoids which satisfy a set of inequations $t \leq t^{\prime}$, for certain bimonoid terms $t, t^{\prime}$. Any collection of ordered bimonoids is contained in a least variety of ordered bimonoids.

One variety of interest is that generated by the language bimonoids. 
Definition 3. Lg denotes the variety of bimonoids generated by the language bimonoids $\mathcal{L}_{\Sigma}$, and $\mathbf{L g} \leq$ denotes the variety of ordered bimonoids generated by the ordered bimonoids of languages $\left(\mathcal{L}_{\Sigma}, \subseteq\right)$.

Remark 4. The variety $\mathbf{L g}_{\leq}$is generated by the ordered bimonoids of finite languages $\mathcal{F}_{\Sigma}$ and thus by the the ordered bimonoids $\mathcal{R}_{\Sigma}$ of regular languages [4].

Labeled posets with serial and parallel product form another example of a bimonoid. An $A$-labeled poset $P=(P, \leq, \ell)$ consists of a poset $\left(P, \leq_{P}\right)$, sometimes written just $(P, \leq)$, and an assignment of an element $v \ell$ in $A$ to each vertex $v$ in $P$. (Here $P$ denotes both the poset and the underlying set of elements or "vertices" of $P$. Thus the expression " $v \in P$ " is meaningful.) When $A$ is understood, we will say only "labeled poset". A morphism $f: P \rightarrow Q$ of $A$-labeled posets is a function $P \rightarrow Q$ which preserves the ordering and the labeling. We agree to identify isomorphic labeled posets, without further mention. (Many authors call an isomorphism class of a labeled poset a "pomset".) To save space, we assume "poset" means "finite poset". We denote the empty poset by 1 . Two operations on labeled posets are important here, sequential, or serial product $P \cdot Q$ and parallel or shuffle product $P \otimes Q$. Given labeled posets $P, Q$, with disjoint underlying sets,

where for $v, v^{\prime} \in P \cup Q$,

$$
\begin{aligned}
P \cdot Q & :=\left(P \cup Q, \leq_{P \cdot Q}\right) \\
P \otimes Q & :=(P \cup Q, \leq P \otimes Q),
\end{aligned}
$$

$$
\begin{array}{r}
v \leq_{P \cdot Q} v^{\prime} \Leftrightarrow v \leq_{P} v^{\prime} \text { or } v \leq_{Q} v^{\prime} \text { or } \\
v \in P \text { and } v^{\prime} \in Q . \\
v \leq_{P \otimes Q} v^{\prime} \Leftrightarrow v \leq_{P} v^{\prime} \text { or } v \leq_{Q} v^{\prime} .
\end{array}
$$

The labeling is extended to $P \otimes Q$ and $P \cdot Q$ in the obvious way.

Definition 5. We let $\mathrm{SP}_{A}$, for "series-parallel" or "shuffle product", denote the least class of posets containing the empty poset 1 , the singleton posets $a$, labeled $a$, for each $a \in A$, closed under the operations $P \cdot Q, P \otimes Q$. The posets in $\mathrm{SP}_{A}$ will be called "series-parallel" posets. We let $\operatorname{SP}(A)$ be the following bimonoid:

$$
\mathbf{S P}(A):=\left(\mathrm{SP}_{A}, \cdot, \otimes, \mathbf{1}\right) \text {. }
$$

Remark 6. Grabowski [8] and Valdes [10] found the following "N-condition" to characterize the series-parallel posets. A poset $P$ is series-parallel iff there is no four-element subset $\{a, b, c, d\} \subseteq P$ whose only nontrivial order relationships are given by

$$
a<c, \quad a<d, \quad b<d .
$$

A nice proof of this fact is given in [7]. It follows from this characterization that any subset of a series-parallel poset, ordered with the inherited order, is itself series-parallel. This fact will be used several times without mention. 
Theorem 7. [7] $\mathbf{S P}(A)$ is freely generated in the variety of all bimonoids by the set $A$.

We will impose an ordering on $\mathbf{S P}(A)$ making use of the alphabet $\Sigma(A)$, defined below. Given the set $A$, let $\bar{A}$ be a set disjoint from $A$, and let

$$
a \mapsto \bar{a}
$$

be a bijection. Define the sets $A_{N}$ and $\bar{A}_{N}$ by:

$$
\begin{aligned}
& A_{N}:=A \times \mathbf{N} \\
& \bar{A}_{N}:=\bar{A} \times \mathbf{N}
\end{aligned}
$$

where $\mathbf{N}=\{0,1, \ldots\}$. We denote elements in $A_{N}$ as $a_{i}, b_{j}$, etc., and elements in $\bar{A}_{N}$ as $\bar{a}_{i}, \bar{b}_{j}$. Let $\Sigma(A)$ be the infinite alphabet

$$
\Sigma(A):=A_{N} \cup \bar{A}_{N} .
$$

Let $h_{0}: \mathbf{S P}(A) \rightarrow \mathcal{L}_{\Sigma(A)}$ be the unique bimonoid morphism satisfying

$$
a h_{0}:=\left\{a_{1} \bar{a}_{1}, a_{2} \bar{a}_{2} \ldots, a_{n} \bar{a}_{n}, \ldots\right\},
$$

each $a \in A$.

Remark 8. An expansion of a poset $P \in \mathbf{S P}(A)$ is a poset $P^{\prime}$ in $\operatorname{SP}(\Sigma(A))$ obtained from $P$ by replacing each vertex $v$ of $P$ by a two element chain $v(1)<$ $v(2)$. The ordering in $P^{\prime}$ is defined as follows:

$$
v(i) \leq v^{\prime}(j) \Leftrightarrow v<v^{\prime} \text { in } P \text { or } v=v^{\prime} \text { and } i \leq j \in[2] \text {. }
$$

If $v$ is labeled $a$ in $P$, then for some $i \geq 1, v(1)$ is labeled $a_{i}$ and $v(2)$ is labeled $\bar{a}_{i}$. Suppose that

$$
v_{1} v_{2} \ldots v_{m}
$$

is a topological run of $P^{\prime}$ (i.e., a surjection $v:[m] \rightarrow P$ such that $v_{i} \leq_{P} v_{j} \Rightarrow$ $i \leq j)$. The trace of this run is the word in $\Sigma(A)^{*}$ formed by concatenating the labels of the vertices in the run. In [4] it is shown that for any poset $P \in \operatorname{SP}(A)$, a word $u$ belongs to $P h_{0}$ iff $u$ is a trace of an expansion of $P$.

One of the main results of [4] is the following.

Theorem 9. The morphism $h_{0}$ is injective. It follows that $\mathbf{L g}$ is the variety of all bimonoids.

For any labeled poset $P$, the words $u$ in $P h_{0}$ satisfy at least the following conditions:

(i) $|u|_{a}=|u|_{\bar{a}}$, for each $a \in A$. ( $\left(|u|_{a}\right.$ is the number of occurrences of the letter $a$ in the word $u$.)

(ii) If $w$ is a prefix of $u,|w|_{a} \geq|w|_{\bar{a}}$, for each $a \in A$. 
We say a word in $\Sigma(A)^{*}$ is balanced if it satisfies both conditions (i) and (ii).

Definition 10. A monoid endomorphism $\varphi$ of $\left(A_{N} \cup \bar{A}_{N}\right)^{*}$ is admissible if, for each $a \in A, i \in \mathbf{N}$, there is some $j \in \mathbf{N}$ with

$$
\begin{aligned}
& a_{i} \varphi=a_{j}, \quad \text { and } \\
& \bar{a}_{i} \varphi=\bar{a}_{j} .
\end{aligned}
$$

Thus, admissible morphisms only change subscripts on letters. For example, an admissible morphism may identify $a_{1}$ and $a_{2}$.

Definition 11. The preorder $\subseteq$ on the set of (balanced) words in $\Sigma(A)^{*}$ is the least reflexive and transitive relation which satisfies the following conditions. $u \sqsubseteq u^{\prime}$ if, for some words $w, w^{\prime}$ and distinct letters $a_{i}, b_{j}$,

$$
\begin{aligned}
& u=w a_{i} b_{j} w^{\prime} \text { and } u^{\prime}=w b_{j} a_{i} w^{\prime} ; \quad \text { or } \\
& u=w \bar{a}_{i} \bar{b}_{j} w^{\prime} \text { and } u^{\prime}=w \bar{b}_{j} \bar{a}_{i} w^{\prime} ; \text { or } \\
& u=w \bar{a}_{i} b_{j} w^{\prime} \text { and } u^{\prime}=w b_{j} \bar{a}_{i} w^{\prime} ; \text { or } \\
& u=u^{\prime} \varphi, \quad \text { for some admissible } \varphi .
\end{aligned}
$$

Definition 12. For $A$-labeled posets $P, Q$ in $\operatorname{SP}(A), P \leq Q$ iff for each word $u \in P h_{0}$ there is a word $u^{\prime} \in Q h_{0}$ with $u \sqsubseteq u^{\prime}$. We call the order $\leq$ the trace ordering on $\mathbf{S P}(A)$ (see Remark 8).

We note some easy consequences of Definition 12.

Proposition 13. For posets $P, Q \in \mathbf{S P}(A)$, the following are equivalent.
1. $P \leq Q$
2. $P h_{0} \subseteq Q h_{0}$.

Since $h_{0}$ is injective, the relation $\leq$ is a partial order on $\mathbf{S P}(A)$.

Remark 14. If $u, v$ are words and $v \in P h_{0}$, for some $P \in \mathbf{S P}(A)$, and if $u \sqsubseteq v$, then $u \in P h_{0}$. It follows that for posets $P, Q \in \mathbf{S P}(A), P \leq Q$ iff for each word $u$ which is maximal in $P h_{0}$ there is some word $v$ which is maximal in $Q h_{0}$ with $u \sqsubseteq v$. Note that by the last clause in Definition 11, each letter occurs at most once in any maximal word.

Proposition 15. Suppose that in $\operatorname{SP}(A), P \leq P_{1}$ and $Q \leq Q_{1}$. Then

$$
P \cdot Q \leq P_{1} \cdot Q_{1} \text { and } P \otimes Q \leq P_{1} \otimes Q_{1} \text {. }
$$

Thus, $(\mathbf{S P}(A), \leq)$ is an ordered bimonoid in $\mathbf{L g}_{\leq}$. 
Theorem 16. [4] For each set $A,(\operatorname{SP}(A), \leq)$ is the free ordered bimonoid in the variety of ordered bimonoids $\mathbf{L g} \leq$.

For later use, we note several other properties of the trace order.

Lemma 17. Suppose that $P \leq Q$ in $\operatorname{SP}(A)$. Then

1. For each $a \in A$, the number of vertices of $P$ labeled $a,|P|_{a}$, is the same as $|Q|_{a}$;

2. The number of minimal elements in $Q$ is at least the number of minimal elements in $P$;

3. The number of maximal elements in $Q$ is at least the number of maximal elements in $P$;

4. The height of $P$ is at least the height of $Q$;

5. The width of $Q$ is at least the width of $P$.

\section{A Metatheorem}

In this section, we present a method to show that the variety $\mathbf{L g}_{\leq}$of ordered bimonoids defined above is not finitely axiomatizable. The method depends upon finding a sequence of posets $P_{n}, P_{n}^{\prime}$ satisfying certain properties.

Suppose that $A$ is the singleton set $\{a\}$.

Theorem 18. Suppose that for each integer $n \geq 1$ there are posets $P_{n}$ and $P_{n}^{\prime}$ in $\mathbf{S P}(A)$ with the following properties:

(P1) $P_{n}^{\prime}$ is a successor of $P_{n}$ with respect to the trace ordering, so that $P_{n}<P_{n}^{\prime}$ and for all $P$ in $\operatorname{SP}(A), P_{n} \leq P<P_{n}^{\prime}$ implies $P=P_{n}$.

(P2) $\left|P_{n}\right|>n$.

(P3) $P_{n}^{\prime}$ does not have a nontrivial series decomposition.

(P4) There are no nonempty posets $U \leq U^{\prime}, V \leq V^{\prime}$ with $P_{n}=U \otimes V$ and $P_{n}^{\prime}=U^{\prime} \otimes V^{\prime}$.

(P5) If

is a valid inequation in $\mathbf{L g}_{\leq}$and

$$
t\left(x_{1}, \ldots, x_{k}\right) \leq t^{\prime}\left(x_{1}, \ldots, x_{k}\right)
$$

$$
\begin{aligned}
& P_{n}=t\left(R_{1}, \ldots, R_{k}\right) \\
& P_{n}^{\prime}=t^{\prime}\left(R_{1}, \ldots, R_{k}\right),
\end{aligned}
$$

for some posets $R_{1}, \ldots, R_{k}$ in $\operatorname{SP}(A)$, then $|t|>n$. 
Then the variety $\mathbf{L}_{\leq}$is not finitely axiomatizable.

The number $|t|$ in condition (P5) denotes the number of occurrences of variables in $t$. Note that since $\mathbf{L g}_{\leq} \vDash t \leq t^{\prime}$, it follows that for each variable $x_{i}$, the number of occurrences of $x_{i}$ in $t$ is the same as the number of occurrences of $x_{i}$ in $t^{\prime}$. Thus $|t|=\left|t^{\prime}\right|$. Note also that condition (P2) follows from (P5) and (P1): Let $t(x), t^{\prime}(x)$ be terms such that $t(a)=P_{n}$ and $t^{\prime}(a)=P_{n}^{\prime}$.

The remainder of this section is devoted to the proof of Theorem 18.

Let $E$ be a finite set of inequations between bimonoid terms with $\mathbf{L g}_{\leq} \vDash E$. Let

$$
n:=\max \left\{|t|,\left|t^{\prime}\right|: t \leq t^{\prime} \in E\right\}
$$

and $m:=\left|P_{n}\right|$. Using the properties of the posets, we construct an ordered bimonoid $B_{E}$ and terms $p(x)$ and $p^{\prime}(x)$ such that:

1. $B_{E}=E$;

2. $B_{E} \not \neq p(x) \leq p^{\prime}(x)$;

3. $\mathbf{L g}_{\leq} \vDash p(x) \leq p^{\prime}(x)$.

To construct $B_{E}$, consider the free bimonoid $\mathbf{S P}(A)$. The relation $\sim$ defined by

$$
P \sim Q \Leftrightarrow|P|,|Q|>m \text { or } P=Q,
$$

is a bimonoid congruence relation on $\mathbf{S P}(A)$. The quotient $\mathbf{S P}(A) / \sim$ may be conveniently represented as the bimonoid whose elements are the labeled posets $P \in \mathbf{S P}(A)$ with $|P|$ at most $m$, and the element 0 which represents the congruence class of the posets $P$ with $|P|>m$. The operations are defined in a straightforward manner.

Now we introduce a partial order on the bimonoid $\mathbf{S P}(A) / \sim$. For $P, Q$ in $\operatorname{SP}(A) / \sim$, we define

$$
P \sqsubseteq Q
$$

iff $P=Q$, or $P, Q \neq 0, P \leq Q$, and either $P \neq P_{n}$ or $Q \neq P_{n}^{\prime}$. Thus, for nonzero elements we keep the ordering in $\operatorname{SP}(A)$, except $P_{n}$ and $P_{n}^{\prime}$ become unrelated. The element 0 is not comparable to any other element. Clearly, $\subseteq$ is reflexive and antisymmetric.

Proposition 19. If $P \sqsubset Q \sqsubset R$, then $P \sqsubset R$. Thus, $\subseteq$ is a partial order.

Proof. The only possible concern is $P=P_{n}$ and $R=P_{n}^{\prime}$. But this is impossible, since $P_{n}^{\prime}$ is a successor of $P_{n}$ in the trace ordering.

The resulting structure, which we denote $B_{E}$, is an ordered bimonoid, since the fact that the operations are monotonic is immediate from (P3) and (P4).

Claim: $B_{E}$ satisfies the inequations in $E$. 
Suppose

$$
t\left(x_{1}, \ldots, x_{k}\right) \leq t^{\prime}\left(x_{1}, \ldots, x_{k}\right)
$$

is in $E$. Since $\operatorname{Lg}_{\leq} \vDash E$, the variables which actually appear in $t$ are the same as that appearing in $t^{\prime}$. We may assume that these are the variables $x_{1}, \ldots, x_{k}$. Moreover, for each $x_{i}$, the number of occurrences of $x_{i}$ in $t$ is the same as the number of occurrences of $x_{i}$ in $t^{\prime}$, in notation: $|t|_{x_{5}}=\left|t^{\prime}\right|_{x_{z}}$. We need to show that

$$
t\left(R_{1}, \ldots, R_{k}\right) \sqsubseteq t^{\prime}\left(R_{1}, \ldots, R_{k}\right),
$$

for all $R_{1}, \ldots, R_{k}$ in $B_{E}$. But the left hand side is 0 iff the right hand side is 0 . If the two sides are nonzero, then the $R_{i}^{\prime} s$ are nonzero, and the two sides are evaluated in $B_{E}$ in the same way as in $\mathbf{S P}(A)$. Thus,

$$
t\left(R_{1}, \ldots, R_{k}\right) \leq t^{\prime}\left(R_{1}, \ldots, R_{k}\right) .
$$

But, by (P5) and the choice of the integer $m$, it is not possible that the left hand side is $P_{n}$ and the right hand side is $P_{n}^{\prime}$, proving (1).

Claim: There is some inequation $p(x) \leq p^{\prime}(x)$ which holds in $L_{g_{<}}$but not in $B_{E}$. Indeed, let $p(x)$ and $p^{\prime}(x)$ be terms with $p(a)=P_{n}$ and $p^{\prime}(a)=P_{n}^{\prime}$ in $\mathbf{S P}(A)$. Since $P_{n} \leq P_{n}^{\prime}$ in $\mathbf{S P}(A), p(x) \leq p^{\prime}(x)$ holds in $\mathbf{L g}_{\leq}$, but fails in $B_{E}$, by construction.

Thus we have proved: If there are posets $P_{n}, P_{n}^{\prime}$ satisfying conditions (P1)-(P5), then for each finite set $E$ of inequations which are valid in $\mathbf{L g}_{\leq}$there exists a finite ordered bimonoid $B_{E}$ and a unary inequation $p(x) \leq p^{\prime}(x)$ which holds in $\mathbf{L g}_{\leq}$but not in $B_{E}$.

Remark 20. It follows easily from the compactness theorem that there is no finite first order axiomatization of $\mathbf{L g}_{\leq}$either.

\section{The Construction}

In this section, we define the sequence of posets $P_{n}$ and $P_{n}^{\prime}$ and prove a number of facts about the ordering of the posets in $\operatorname{SP}(A)$ in order to show the sequence has the properties (P1)-(P5) of Theorem 18. All posets are assumed to belong to $\operatorname{SP}(A)$, where $A$ is a singleton set, say $A=\{a\}$.

The collection of posets in $\mathbf{S P}(B)$ with a unique maximal vertex and a unique minimal vertex is denoted $\mathbf{S P}_{1}(B)$.

For any poset $R \in \mathbf{S P}(B)$, where $B$ contains the letter $a$, define the "top-bottom" of $R, t b(R)$ as follows:

$$
t b(R):=a \cdot R \cdot a .
$$

Thus, $t b(R)$ has a unique maximal and minimal element. 
Definition 21. Define the following sequence of posets in $\operatorname{SP}(A)$.

$$
\begin{aligned}
& P_{1}=a \cdot a \\
& P_{1}^{\prime}=a \otimes a .
\end{aligned}
$$

Assume $P_{n}, P_{n}^{\prime}$ defined so that $P_{n}^{\prime}$ is a successor of $P_{n}$.

$$
\begin{aligned}
& P_{n+1}:=\left(a \cdot t b\left(P_{n}\right)\right) \otimes\left(a \cdot t b\left(P_{n}\right)\right) \otimes\left(a \cdot t b\left(P_{n}^{\prime}\right)\right) \\
& P_{n+1}^{\prime}:=a \cdot\left(t b\left(P_{n}\right) \otimes t b\left(P_{n}^{\prime}\right)\right) \otimes(a \otimes a) \cdot t b\left(P_{n}^{\prime}\right) .
\end{aligned}
$$

The fact that the posets $P_{n}, P_{n}^{\prime}$ satisfy properties (P2),(P3) and (P4) of Theorem 18 is either immediate or follows easily from Lemma 17. Thus, it remains to establish properties (P1) and (P5).

It is clear that $P_{1}^{\prime}$ is the unique successor of $P_{1}$. ¿From Lemma 30 below, it follows that $P_{n}^{\prime}$ is a successor of $P_{n}$, for all $n \geq 1$.

Suppose that $R$ is a poset in $\mathbf{S P}(A)$ having the minimal elements $x_{1}, x_{2}, \ldots$. Define the subposet $R_{i}$ to consist of those vertices above only the one minimal vertex $x_{i}$ :

$$
R_{i}:=\left\{v \in R: x_{j} \leq v \Rightarrow j=i\right\}
$$

Lemma 22. The subposet $R_{i}$ of $R$ contains a maximal element of $R$ iff

$$
R=R_{i} \otimes\left(R-R_{i}\right) .
$$

Proof. Clearly, if $R=R_{i} \otimes\left(R-R_{i}\right)$, then any vertex maximal in $R_{i}$ is maximal in $R$. We prove the converse. Suppose that $R_{i}$ contains the maximal vertex $y_{i}$. If $v \notin R_{i}$, then $v \geq x_{j}$ for some $j \neq i$. Thus for any $v^{\prime} \in R_{i}$, it is impossible that $v^{\prime} \geq v$. Conversely, if $v \geq v^{\prime}$, the subposet of $R$ consisting of $y_{i}, x_{i}, v, x_{j}$ shows that $R$ violates the "N-condition", and cannot belong to $\operatorname{SP}(A)$. (See Remark 6 for the "N-condition".)

Lemma 23. Suppose that the posets $P_{i}, Q_{i}, i \in[3]$, all belong to $\mathbf{S P}_{1}(A)$ and each has $N \geq 1$ vertices. Let $P:=\otimes_{i=1}^{3} P_{i}, Q:=\otimes_{i=1}^{3} Q_{i}$. If

$$
P \leq R \leq Q,
$$

then there are posets $R_{i}, i \in[3]$ in $\mathbf{S P}_{1}(A)$ such that $R=\otimes_{i=1}^{3} R_{i}$, and each $R_{i}$ has size $N$.

Proof. We may assume $N>1$ or else $P=R=Q$. Now by Lemma 17, $R$ has 3 minimal and 3 maximal vertices. Suppose that $\left\{x_{1}, x_{2}, x_{3}\right\}$ are the minimal vertices and that $\left\{y_{1}, y_{2}, y_{3}\right\}$ are the maximal vertices in $R$. The poset $R$ has no isolated vertex. (Indeed, if $S, T$ are any posets in $\operatorname{SP}(A)$ such that $a \otimes S \leq T$ then $T=a \otimes T^{\prime}$, for some $T^{\prime} \in \mathbf{S P}(A)$.) Thus, the vertices $x_{i}, y_{j}$ are all distinct. For an element $i \in$ [3], the set $R_{i}$ was defined in (7). 
Sublemma24. - Some set $R_{i}$ has at least $N$ vertices.

- No set $R_{i}$ can have more than $N$ vertices.

- One of the sets $R_{i}$ which has exactly $N$ vertices contains one of the maximal vertices $y_{j}$.

- None of the sets $R_{i}$ can contain 2 maximal vertices.

Proof. We show first that some set $R_{i}$ has at least $N$ vertices. Indeed, the word

$$
u:=a_{1}^{3} \bar{a}_{1}\left(a_{2} \bar{a}_{2}\right)^{N-1} \bar{a}_{1}\left(a_{2} \bar{a}_{2}\right)^{N-1} \bar{a}_{1}\left(a_{2} \bar{a}_{2}\right)^{N-1}
$$

belongs to $P h_{0}$ and hence to $R h_{0}$. The vertices in $R$ of any run with trace $u$ (see Remark 8) corresponding to the first occurrence of $\bar{a}_{1}$ and the first $N-1 a_{2}$ 's belong to some set $R_{i}$. Hence this $R_{i}$ has at least $N$ vertices.

But no set $R_{i}$ can have more than $N$ vertices. Otherwise, there is a word

$$
a_{1}^{3} \bar{a}_{1}\left(a_{2} \bar{a}_{2}\right)^{k} w
$$

in $R h_{0}$ where $k \geq N$, but there is no such word in $Q h_{0}$. Thus, some set $R_{i}$ contains exactly $N$ vertices.

One of the sets $R_{i}$ which has exactly $N$ vertices must contain one of the maximal vertices $y_{j}$. Indeed, otherwise, the word

$$
a_{1}^{3} \bar{a}_{1}\left(a_{2} \bar{a}_{2}\right)^{N-2} a_{3} \bar{a}_{1}\left(a_{2} \bar{a}_{2}\right)^{N-2} a_{3} \bar{a}_{1}\left(a_{2} \bar{a}_{2}\right)^{N-2} a_{3} \bar{a}_{3}^{3}
$$

does not belong to $R h_{0}$ even though it does belong to $P h_{0}$.

Now we show none of the sets $R_{i}$ can contain 2 maximal vertices. Indeed, if $\left|R_{i}\right|=k+3 \leq N$ and $R_{i}$ contains two of the $y_{j}$, then there is a word

$$
a_{1}^{3} \bar{a}_{1}\left(a_{2} \bar{a}_{2}\right)^{k} a_{3} a_{3} v \bar{a}_{3} \bar{a}_{3}
$$

in $R h_{0}$, where $v$ does not contain $\bar{a}_{3}$. But there is no such word in $Q h_{0}$.

Now suppose that $R_{1}$ has $N$ vertices and contains the maximal element $y_{1}$, say. Then by Lemma 22,

$$
R=R_{1} \otimes\left(R-R_{1}\right) .
$$

Let $R^{\prime}=\left(R-R_{1}\right)$. We now show $R^{\prime}=R_{2} \otimes R_{3}$. Note that $R_{2} \cup R_{3} \subseteq R^{\prime}$. We know $R^{\prime}$ contains the two maximal vertices $y_{2}, y_{3}$ and the two minimal vertices $x_{2}, x_{3}$. Suppose, without loss of generality, that $y_{2}>x_{2}$. Then it is impossible that $y_{2}>x_{3}$. Otherwise, if $y_{3}>x_{2}$ (or $y_{3}>x_{3}$ ), the "N-condition" implies that both $y_{2}$ and $y_{3}$ are greater than both $x_{2}$ and $x_{3}$. But then, the word $u$ of (8) is not in $R h_{0}$ even though it belongs to $P h_{0}$. Thus, $y_{2} \in R_{2}$, showing that

$$
R^{\prime}=R_{2} \otimes\left(R^{\prime}-R_{2}\right)
$$

by Lemma 22 . But $R_{3}=R^{\prime}-R_{2}$, since $R$ has only 3 minimal vertices. Thus,

$$
R=R_{1} \otimes R_{2} \otimes R_{3} \text {. }
$$

Since $\left|R_{1}\right|=N$, and $\left|R_{2}\right|,\left|R_{3}\right| \leq N$ and $|R|=3 N$, it follows that $\left|R_{2}\right|=\left|R_{3}\right|=$ $N$, completing the proof of Lemma 23 . 
Remark 25. Lemma 23 holds when 3 is replaced by any positive integer.

Lemma 26. Suppose that $Q$ is a successor of $P$, where $P, Q \in \operatorname{SP}_{1}(A)$, and that $R_{1}, R_{2}, R_{3}$ are posets in $\mathbf{S P}_{1}(A)$ with $|P|=|Q|=\left|R_{1}\right|=\left|R_{2}\right|=\left|R_{3}\right|$. If

$$
P \otimes P \otimes Q \leq R_{1} \otimes R_{2} \otimes R_{3} \leq P \otimes Q \otimes Q,
$$

then, for each $i \in[3], R_{i} \in\{P, Q\}$ and either exactly one or exactly two of the $R_{i}$ are equal to $P$.

Proof. First we show $P \leq R_{i}$, for each $i \in$ [3]. Otherwise, there is a maximal word $a_{1} \bar{a}_{1} u a_{2} \bar{a}_{2}$ in $P h_{0}$ which is not in $R_{i} h_{0}$. Then

$$
a_{1}^{3} \bar{a}_{1} u a_{2} \bar{a}_{2} \bar{a}_{1} u a_{2} \bar{a}_{2} \bar{a}_{1} u a_{2} \bar{a}_{2}
$$

is in $(P \otimes P \otimes Q) h_{0}$ but not in $\left(R_{1} \otimes R_{2} \otimes R_{3}\right) h_{0}$.

Now we show $R_{i} \leq Q$, for each $i \in[3]$. Otherwise, choose a word $a_{1} \bar{a}_{1} u a_{2} \bar{a}_{2}$ in $R_{i} h_{0}$ not in $Q h_{0}$ such that $a_{1}, \bar{a}_{1}$ do not occur in $u$. Since $P \leq Q$, this word does not occur in $P h_{0}$ either. Then there is a word

$$
a_{1}^{3} \bar{a}_{1} u a_{2} \bar{a}_{2} v
$$

in $\left(R_{1} \otimes R_{2} \otimes R_{3}\right) h_{0}$ not in $(P \otimes Q \otimes Q) h_{0}$.

Since $Q$ is a successor of $P$, it follows that either two of the $R_{i}$ are $P$ or exactly one $R_{i}$ is $P$.

Corollary 27. Suppose that $P, Q \in \mathbf{S P}_{1}(A)$. If $Q$ is a successor of $P$, then $P \otimes Q \otimes Q$ is a successor of $P \otimes P \otimes Q$.

Proof. Suppose that

$$
P \otimes P \otimes Q \leq R \leq P \otimes Q \otimes Q .
$$

By Lemma 23, $R=R_{1} \otimes R_{2} \otimes R_{3}$, where $P, Q$ and the $R_{i}$ satisfy the hypotheses of Lemma 26. Hence, by that Lemma, $R=P \otimes Q \otimes Q$ or $R=P \otimes Q \otimes Q$.

Lemma 28. Suppose that $P, Q$ belong to $\mathbf{S P}(A)$, and that $Q$ is a successor of $P$. Then $t b(Q)$ is a successor of $t b(P)$, and both $t b(P)$ and $t b(Q)$ belong to $\mathbf{S P}_{1}(A)$.

Lemma 29. Suppose that $P, Q$ belong to $\mathbf{S P}(A)$ and that $P \leq Q$. Let $P^{\prime}, Q^{\prime}$ be defined by

$$
\begin{aligned}
& P^{\prime}:=(a \cdot P) \otimes(a \cdot P) \otimes(a \cdot Q) \\
& Q^{\prime}:=(a \cdot(P \otimes Q)) \otimes((a \otimes a) \cdot Q)
\end{aligned}
$$

Then $P^{\prime} \leq Q^{\prime}$. 
Proof. Up to an admissible bijection, each maximal word in $P^{\prime} h_{0}$ is in either $U_{1}$ or $U_{2}$, where $K=P h_{0}$ and $L=Q h_{0}$ and where

$$
\begin{aligned}
& U_{1}:=a_{1} a_{2} a_{3} \bar{a}_{1}\left(K \otimes \bar{a}_{2} K \otimes \bar{a}_{3} L\right) \\
& U_{2}:=a_{1} a_{2} a_{3} \bar{a}_{1}\left(L \otimes \bar{a}_{2} K \otimes \bar{a}_{3} K\right) .
\end{aligned}
$$

But, $K \subseteq L$, so that

$\left(K \otimes \bar{a}_{2} K \otimes \bar{a}_{3} L\right) \subseteq\left(K \otimes \bar{a}_{2} L \otimes \bar{a}_{3} L\right)$.

The inequation

$$
(x \cdot z) \otimes(y \cdot z) \leq z \otimes((x \otimes y) \cdot z)
$$

is valid in $\mathbf{S P}(A)$ (see [7]). Hence,

$\left(K \otimes \bar{a}_{2} L \otimes \bar{a}_{3} L\right) \subseteq\left(K \otimes L \otimes\left(\bar{a}_{2} \otimes \bar{a}_{3}\right) L\right)$.

Thus,

$$
\begin{aligned}
U_{1} & \subseteq a_{1} a_{2} a_{3} \bar{a}_{1}\left(K \otimes L \otimes\left(\bar{a}_{2} \otimes \bar{a}_{3}\right) L\right) \\
& \subseteq Q^{\prime} h_{0} .
\end{aligned}
$$

Similarly,

$$
\begin{aligned}
L \otimes \bar{a}_{2} K \otimes \bar{a}_{3} K & \subseteq L \otimes K \otimes\left(\bar{a}_{2} \otimes \bar{a}_{3}\right) K \\
& \subseteq K \otimes L \otimes\left(\bar{a}_{2} \otimes \bar{a}_{3}\right) L
\end{aligned}
$$

so that

$$
\begin{aligned}
U_{2} & \subseteq a_{1} a_{2} a_{3} \bar{a}_{1}\left(K \otimes L \otimes\left(\bar{a}_{2} \otimes \bar{a}_{3}\right) L\right) \\
& \subseteq Q^{\prime} h_{0} .
\end{aligned}
$$

Lemma 30. Suppose that $P, Q$ belong to $\operatorname{SP}_{1}(A)$ and that $Q$ is a successor of $P$. Let $P^{\prime}, Q^{\prime}$ be defined by

$$
\begin{aligned}
& P^{\prime}:=(a \cdot P) \otimes(a \cdot P) \otimes(a \cdot Q) \\
& Q^{\prime}:=(a \cdot(P \otimes Q)) \otimes((a \otimes a) \cdot Q) .
\end{aligned}
$$

Then $Q^{\prime}$ is a successor of $P^{\prime}$

Proof. Lemma 29 showed that $P^{\prime} \leq Q^{\prime}$. Suppose that

$$
P^{\prime} \leq R \leq Q^{\prime} \text {. }
$$

$R$ must have 3 minimal vertices. Let $R_{1}$ denote the poset in $\mathbf{S P}(A)$ obtained from $R$ by deleting the minimal vertices. Let $P_{1}:=P \otimes P \otimes Q$ and $Q_{1}:=P \otimes Q \otimes Q$.

We claim that $P_{1} \leq R_{1} \leq Q_{1}$. Indeed, if $R_{1} \notin Q_{1}$, there is a word $u \in R_{1} h_{0}-$ $Q_{1} h_{0}$. Then $a_{1}^{3} \bar{a}_{1}^{3} u \in R h_{0}-Q^{\prime} h_{0}$, a contradiction. Similarly, $P_{1} \leq R_{1}$. But, since $Q_{1}$ is a successor of $P_{1}$ by Lemma 27, it follows that $R_{1}=P_{1}$ or $R_{1}=Q_{1}$. Thus, $R_{1}$ has 3 connected components, each having a unique minimal element.

Suppose that the minimal elements of $R_{1}$ are $y_{1}, y_{2}, y_{3}$. But $R$ itself has three minimal elements, say $x_{1}, x_{2}, x_{3}$. Each $y_{i}$ is a successor of at least one $x_{j}$. Up 
to isomorphism, there are only two possible posets determined by the order relations among the $x_{i}$ 's and $y_{j}$ 's:

$$
\left(x_{1} \cdot y_{1}\right) \otimes\left(x_{2} \cdot y_{2}\right) \otimes\left(x_{3} \cdot y_{3}\right)
$$

or

$$
\left(\left(x_{1} \otimes x_{2}\right) \cdot y_{1}\right) \otimes\left(x_{3} \cdot\left(y_{2} \otimes y_{3}\right)\right) .
$$

Indeed, if, for example, the ordering were

$$
\left(\left(x_{1} \otimes x_{2}\right) \cdot\left(y_{1} \otimes y_{2}\right)\right) \otimes\left(x_{3} \cdot y_{3}\right),
$$

then the word

$$
a_{1}^{3} \bar{a}_{1}\left(a_{2} \bar{a}_{2}\right)^{K} \bar{a}_{1}\left(a_{2} \bar{a}_{2}\right)^{K} \bar{a}_{1}\left(a_{2} \bar{a}_{2}\right)^{K}
$$

would be in $P^{\prime} h_{0}-R h_{0}$, where $K=|P| \geq 2$.

Note that by the "N-condition" (see Remark 6), in case (9) it is impossible that $x_{i}$ is below any vertex above $x_{j}$, when $i \neq j$. In case (10), $x_{3}$ is not below any vertex above both $x_{1}$ and $x_{2}$, and neither $x_{1}$ nor $x_{2}$ is below any vertex above $x_{3}$.

We now consider the two cases separately.

Case (9). In this case, either

$$
\begin{aligned}
& R=(a \cdot P) \otimes(a \cdot P) \otimes(a \cdot Q)=P^{\prime}, \text { or } \\
& R=(a \cdot P) \otimes(a \cdot Q) \otimes(a \cdot Q) .
\end{aligned}
$$

We show that (12) is impossible. Indeed, if $v$ is a word in $Q h_{0}-P h_{0}$ and $u$ is a word in $P h_{0}$, neither of which contain the letters $a_{1}, \bar{a}_{1}$, then

$$
a_{1}^{3} \bar{a}_{1} v \bar{a}_{1} v \bar{a}_{1} u \in R h_{0}-Q^{\prime} h_{0} .
$$

Case (10). In this case, either

$$
\begin{aligned}
& R=a \cdot(P \otimes Q) \otimes(a \otimes a) \cdot Q=Q^{\prime}, \quad \text { or } \\
& R=a \cdot(Q \otimes Q) \otimes(a \otimes a) \cdot P, \text { or, } \\
& R=a \cdot(P \otimes Q) \otimes(a \otimes a) \cdot P, \text { or, } \\
& R=a \cdot(P \otimes P) \otimes(a \otimes a) \cdot Q .
\end{aligned}
$$

We show each of the last three possibilities leads to a contradiction. Choose maximal words $v$ in $Q h_{0}-P h_{0}$ and $u \in P h_{0}$, neither of which contain the letters $a_{1}, \bar{a}_{1}$.

(15) and (16) are impossible. Otherwise $P^{\prime} \not R$. Indeed, if (15) holds,

$$
a_{1}^{3} \bar{a}_{1} u \bar{a}_{1} u \bar{a}_{1} v \in P^{\prime} h_{0}-R h_{0} .
$$

And if (16) holds,

$$
a_{1}^{3} \bar{a}_{1} v \bar{a}_{1} u \bar{a}_{1} u \in P^{\prime} h_{0}-R h_{0} .
$$

(14) is impossible: otherwise $R \nsubseteq Q^{\prime}$. Indeed, writing $v=a_{i} \bar{a}_{i} v^{\prime}$,

$$
a_{1}^{3} \bar{a}_{1} a_{i} a_{i} \bar{a}_{i} v^{\prime} \bar{a}_{i} v^{\prime} \bar{a}_{1} \bar{a}_{1} u \in R h_{0}-Q^{\prime} h_{0} \text { 。 }
$$

The proof of Lemma 30 is complete. 
Corollary 31. For each $n \geq 1, P_{n}^{\prime}$ is a successor of $P_{n}$. Thus property (P1) of Theorem 18 is established.

We now establish property (P5) in the following form.

Lemma 32. Suppose that $B$ is any set containing the letter ' $a$ '. Suppose that $Q \leq Q^{\prime} \in \mathbf{S P}(B)$ and $\varphi: \mathbf{S P}(B) \rightarrow \mathbf{S P}(A)$ is an ordered bimonoid morphism such that $Q \varphi=P_{n}$ and $Q^{\prime} \varphi=P_{n}^{\prime}$. Then $|Q|>n$.

Proof. We may assume that $b \varphi \neq 1$, all $b \in B$, and that $b \varphi=a \Rightarrow b=a$. We use induction on $n$. The case $n=1$ is clear. Suppose that $Q \leq Q^{\prime}$ and that $Q \varphi=P_{n+1}$ and $Q^{\prime} \varphi \doteq P_{n+1}^{\prime}$. It is easy to see that

$$
\begin{aligned}
Q & =\left(a \cdot Q_{1}\right) \otimes\left(a \cdot Q_{2}\right) \otimes\left(a \otimes Q_{3}\right) \\
Q^{\prime} & =\left(a \cdot\left(Q_{1}^{\prime} \otimes Q_{2}^{\prime}\right)\right) \otimes\left((a \otimes a) \cdot Q_{3}^{\prime}\right),
\end{aligned}
$$

where

$$
\begin{aligned}
& Q_{i} \varphi=t b\left(P_{n}\right), \quad i=1,2 \quad \text { and } \quad Q_{3} \varphi=t b\left(P_{n}^{\prime}\right) \\
& Q_{1}^{\prime} \varphi=t b\left(P_{n}\right) \quad \text { and } Q_{j}^{\prime} \varphi=t b\left(P_{n}^{\prime}\right), \quad j=2,3 .
\end{aligned}
$$

The posets $Q_{i}, Q_{i}^{\prime}, i \in[3]$, have a unique maximal and minimal element.

Sublemma 33. Suppose that $R_{i}, R_{i}^{\prime}, i=1,2$, are posets in $\mathbf{S P}_{1}(B)$ such that

$$
R_{1} \otimes R_{2} \leq R_{1}^{\prime} \otimes R_{2}^{\prime}
$$

Then either $R_{1} \leq R_{2}^{\prime}$ or $R_{2} \leq R_{2}^{\prime}$.

Proof. In order to obtain a contradiction, assume that $u_{i}$ is a maximal word in $R_{i} h_{0}-R_{2}^{\prime} h_{0}, i=1,2$. We may assume that

$$
\begin{aligned}
& u_{1}=x \bar{x} v_{1} y \bar{y} \\
& u_{2}=x^{\prime} \overline{x^{\prime}} v_{2} y^{\prime} \overline{y^{\prime}}
\end{aligned}
$$

where $x, y, x^{\prime}, y^{\prime}$ are distinct letters in $\Sigma(B)$ and where $v_{1}$ and $v_{2}$ have no letter in common and do not contain the symbols $x, y, x^{\prime}, y^{\prime}, \bar{x}, \bar{y}, \overline{x^{\prime}}, \overline{y^{\prime}}$. Then

$$
x x^{\prime} \bar{x} v_{1} y \overline{x^{\prime}} v_{2} y^{\prime} \bar{y} \bar{y}^{\prime} \in R_{1} h_{0} \otimes R_{2} h_{0}-\left(R_{1}^{\prime} h_{0} \otimes R_{2}^{\prime} h_{0}\right) \text {. }
$$

Note that by symmetry, with the assumptions of Sublemma 33 , also $R_{1} \leq R_{1}^{\prime}$ or $R_{2} \leq R_{1}^{\prime}$.

Sublemma 34. Suppose that $R_{i}, R_{i}^{\prime}, i \in[3]$, belong to $\mathbf{S P}_{1}(B)$. Let

$$
\begin{aligned}
R & :=\left(a \cdot R_{1}\right) \otimes\left(a \cdot R_{2}\right) \otimes\left(a \cdot R_{3}\right) \\
R^{\prime} & :=\left(a \cdot\left(R_{1}^{\prime} \otimes R_{2}^{\prime}\right)\right) \otimes\left((a \otimes a) \cdot R_{3}^{\prime}\right) .
\end{aligned}
$$

Then if $R \leq R^{\prime}$, then

$$
\begin{aligned}
R_{1} \otimes R_{2} & \leq R_{1}^{\prime} \otimes R_{2}^{\prime} \\
R_{3} & \leq R_{3}^{\prime}, \quad \text { and } \\
\left|R_{1}\right| & =\left|R_{2}\right|=\left|R_{3}\right| .
\end{aligned}
$$


Of course, by symmetry, also many more relations hold, e.g.,

$$
\begin{aligned}
R_{1} \otimes R_{3} & \leq R_{1}^{\prime} \otimes R_{2}^{\prime} \\
R_{2} & \leq R_{3}^{\prime} .
\end{aligned}
$$

Proof of Sublemma 34. First, we show $\left|R_{1}\right|+\left|R_{2}\right|=\left|R_{1}^{\prime}\right|+\left|R_{2}^{\prime}\right|$. Let $u_{i} \in R_{i} h_{0}$ be maximal, $i \in[3]$, and assume the three words have no letter in common and do not contain the letter $a_{1}$. Write

$$
u_{i}=u_{i}^{\prime} y_{i} \bar{y}_{i},
$$

where $y_{i}, i \in[3]$, are letters. Since the word

$$
w:=a_{1}^{3} \bar{a}_{1} u_{1}^{\prime} y_{1} \bar{a}_{1} u_{2}^{\prime} y_{2} \bar{a}_{1} u_{3}^{\prime} y_{3} \bar{y}_{1} \bar{y}_{2} \bar{y}_{3}
$$

is in $R h_{0}$ and since $R \leq R^{\prime}$, we know $w \in R^{\prime} h_{0}$. This is possible only if $\left|R_{1}\right|+$ $\left|R_{2}\right|=\left|R_{1}^{\prime}\right|+\left|R_{2}^{\prime}\right|$. By symmetry, $\left|R_{1}\right|+\left|R_{3}\right|=\left|R_{1}^{\prime}\right|+\left|R_{2}^{\prime}\right|$ and $\left|R_{2}\right|+\left|R_{3}\right|=$ $\left|R_{1}^{\prime}\right|+\left|R_{2}^{\prime}\right|$. Hence,

$$
\left|R_{1}\right|=\left|R_{2}\right|=\left|R_{3}\right|=N \text {, say. }
$$

Then, $\left|R_{1}^{\prime}\right|+\left|R_{2}^{\prime}\right|=2 N$ and $\left|R_{3}^{\prime}\right|=N$. But then

$$
R_{1} \otimes R_{2} \leq R_{1}^{\prime} \otimes R_{2}^{\prime} \quad \text { and } \quad R_{3} \leq R_{3}^{\prime} .
$$

Indeed, let $u \in\left(R_{1} \otimes R_{2}\right) h_{0}$ be a word not containing the symbol $a_{1}$. Let $v \in R_{3} h_{0}$ have no letter in common with $u$. Then

$$
w:=a_{1}^{3} \bar{a}_{1} \bar{a}_{1} u \bar{a}_{1} v \in R h_{0},
$$

so that $w \in R^{\prime} h_{0}$. But this can happen only if $u \in\left(R_{1}^{\prime} \otimes R_{2}^{\prime}\right) h_{0}$ and $v \in R_{3}^{\prime} h_{0}$.

Proof of Lemma 32, completed. By Sublemmas 34 and 33, it follows that $Q_{1} \leq Q_{2}^{\prime}$ or $Q_{2} \leq Q_{2}^{\prime}$. Suppose that $Q_{2} \leq Q_{2}^{\prime}$. Since $Q_{2} \varphi=t b\left(P_{n}\right)$ and $Q_{2}^{\prime} \varphi=t b\left(P_{n}^{\prime}\right)$, we have $Q_{2}=t b\left(\bar{Q}_{2}\right)$ and $Q_{2}^{\prime}=t b\left(\bar{Q}_{2}^{\prime}\right)$, where $\bar{Q}_{2} \varphi=P_{n}$ and $\overline{Q_{2}^{\prime}} \varphi=P_{n}^{\prime}$. By the induction assumption, $\left|\bar{Q}_{2}\right|>n$, so that $\left|Q_{2}\right|>n+2$, showing $|Q|>n+1$ 。

Remark 35. With a different argument, we can show that the posets $P_{n}$ and $P_{n}^{\prime}$ satisfy the following stronger version of (P5). For all terms $t\left(x_{1}, \ldots, x_{k}\right)$ and $t^{\prime}\left(x_{1}, \ldots, x_{k}\right)$ such that each variable $x_{i}$ occurs the same number of times in $t$ as in $t^{\prime}$, if $P_{n}=t\left(Q_{1}, \ldots, Q_{k}\right)$ and $P_{n}^{\prime}=t^{\prime}\left(Q_{1}, \ldots, Q_{k}\right)$, for some posets $Q_{j}$ in $\operatorname{SP}(A)$, then $|t|>n$.

\section{Final Remarks}

Although in the last section we formulated most of the lemmas only for the case that $A$ is a singleton, several of them hold for larger alphabets. The assumption that $A$ is a singleton made some proofs notationally simpler.

After proving the metatheorem Theorem 18 , the nontrivial task was to find the actual posets $P_{n}, P_{n}^{\prime}$ satisfying (P1)-(P5). The difficulty was finding the balance 
among the various properties. According to (P1), $P_{n}$ and $P_{n}^{\prime}$ should be close to one another. But, by (P4) and (P5), their structure should be very different. By (P4), they cannot have a parallel decomposition into nontrivial posets related by the trace ordering, again a property that says they are far from one another. The particular construction we used was not forced. In particular, we might have made the following definitions:

$$
\begin{aligned}
& P_{n+1}:=\left((a \otimes a) \cdot t b\left(P_{n}\right)\right) \otimes\left((a \otimes a) \cdot t b\left(P_{n}^{\prime}\right)\right) \\
& P_{n+1}^{\prime}:=\left(a \cdot t b\left(P_{n}^{\prime}\right)\right) \otimes\left((a \otimes a \otimes a) \cdot t b\left(P_{n}^{\prime}\right)\right) .
\end{aligned}
$$

The proof for this choice is similar.

The lemmas proved in Section 4 do not give a full account of the order structure of SP $(A)$, but are sufficient for the present paper. We plan to give a systematic account of this topic, together with some other results, in a future paper.

\section{Acknowledgments}

The second author would like to thank Volker Diekert and the Theory Group at the Institute of Computer Science of the University of Stuttgart for their hospitality. We both thank László Bernátsky for his very careful proof reading.

\section{References}

1. L. Aceto. Full abstraction for series-parallel pomsets. In Proceedings of TAPSOFT '91, volume 493 of Lecture Notes in Computer Science, pages 1-40. Springer-Verlag, 1991.

2. L. Aceto and M. Hennessy. Towards action refinement in process algebras. Information and Computation, 103(2):204-269, 1993.

3. S. L. Bloom. Varieties of ordered algebras. Journal of Computer and System Sciences, 45:200-212, 1976.

4. S. L. Bloom and Z. Ésik. Free Shuffle Algebras in Language Varieties. To appear. Obtainable by anonymous ftp from menger.eecs.stevens-tech.edu in directory pub/bloom/shuffle.

5. Z. Ésik and M. Bertol. Nonfinite axiomatizability of the equational theory of shuffle. To appear.

6. Jay Loren Gischer. Partial Orders and the Axiomatic Theory of Shuffle. PhD thesis, Stanford University, Computer Science Dept., 1984.

7. Jay Loren Gischer. The equational theory of pomsets. Theoretical Computer Science, 61:199-224, 1988.

8. Jan Grabowski. On partial languages. Fundamenta Informatica, IV(2):427-498, 1981.

9. Vaughan Pratt. Modeling concurrency with partial orders. International Journal of Parallel Processing, 15(1):33-71, 1986.

10. J. Valdes, R. E. Tarjan, and E. L. Lawler. The recognition of series-parallel digraphs. SIAM Journal of Computing, 11(2):298-313, 1981. 